\title{
Supply of Healthcare Providers in Relation to County Socioeconomic and Health Status
}

\author{
Matthew A. Davis, MPH, PhD $1,2,3$, Rebecca Anthopolos, $M A^{4}$, Joshua Tootoo, $\mathrm{MS}^{4}$, \\ Marita Titler, RN, PhD², Julie P. W. Bynum, MD, MPH $H^{5}$, and Scott A. Shipman, MD, MPH 5.6
}

${ }^{1}$ Institute for Healthcare Policy and Innovation, University of Michigan, Ann Arbor, MI, USA; ${ }^{2}$ Department of Systems, Populations, and Leadership, University of Michigan School of Nursing, Ann Arbor, MI, USA; ${ }^{3}$ University of Michigan Institute for Social Research, Ann Arbor, MI, USA; ${ }^{4} \mathrm{National}$ Center for Geospatial Medicine, Rice University, Houston, TX, USA; ${ }^{5}$ The Dartmouth Institute for Health Policy and Clinical Practice, Geisel School of Medicine at Dartmouth, Lebanon, NH, USA; ${ }^{\circ}$ Association of American Medical Colleges, Washington, DC, USA.

J Gen Intern Med 33(4):412-4

DOI: $10.1007 / \mathrm{s} 11606-017-4287-4$

(c) Society of General Internal Medicine 2018

\section{INTRODUCTION}

Non-physician clinicians now constitute nearly one-quarter of the US primary care workforce, and as such are expected to play a significant role in offsetting projected physician shortfalls. ${ }^{1,2}$ It has long been recognized that the per capita availability of physicians varies substantially across regions, ${ }^{3}$ and that physicians tend to locate in more affluent locales rather than areas of greatest need. ${ }^{4}$ Less is known about the practice location of non-physician clinicians and whether they exhibit similar patterns to those of physicians. Therefore, we examined the relationship of nurse practitioner and physician assistant supply with local socioeconomic and health status. As an example of a healthcare profession that operates outside the traditional medical system, $80 \%$ of whom operate as small business owners, we also examined the distribution of US chiropractors.

\section{METHODS}

To identify active providers, we linked providers listed in the 2014 National Plan and Provider Enumeration System to Medicare claims. Using practice location and specialty code, we identified the total number of adult primary care physicians (internal medicine, family medicine, and general practice), nurse practitioners, physician assistants, and chiropractors in each US county. Data on county characteristics were gathered from the 2010 US Census, and the presence of a hospital was identified using the Area Health Resource File. To control for the presence of training institutions, we geocoded US professional schools for these provider groups using data from school accreditation bodies. County-level life expectancy was used as a measure of regional health status ${ }^{5}$ and countylevel median household income as a measure of socioeconomic status - both were collapsed into quartiles.

Published online January 23, 2018
To examine the relationship of per capita supply of providers with county socioeconomic and health status level, Poisson regression was used, with the adult population as an offset to adjust for county differences.

\section{RESULTS}

We identified 272,105 primary care physicians, 63,615 nurse practitioners, 47,006 physician assistants, and 43,278 chiropractors who were actively providing care in 2014 (Table 1). The unadjusted rate of providers increased across levels of socioeconomic and health status for all providers except nurse practitioners.

Adjusted for county-level differences, the supply of primary care physicians increased across socioeconomic status ( $p$-trend $=0.01)$. For instance, the number of primary care physicians was $31 \%$ higher in the highest income quartile than in the lowest $(\mathrm{RR}=1.31$ [95\% CI: 1.15, 1.48]; Fig. 1). The distribution of physician assistants exhibited a pattern similar to that of primary care physicians. Supply of chiropractors was most strongly related to county socioeconomic and health status ( $p$ trend $<0.001$ for both), whereas nurse practitioners were the only provider group to exhibit an inverse association between per capita supply and county socioeconomic and health status.

\section{DISCUSSION}

To our knowledge, this study is the first to directly compare the relationship between provider supply and county socioeconomic and health status for this broad set of frontline US healthcare providers. Across all provider groups except nurse practitioners, we found a higher supply of providers in areas of higher income and better health status. Chiropractor supply was most strongly associated with these factors.

There is an extensive body of literature on the value of having access to primary care. ${ }^{6}$ The supply of other providers who might substitute for physicians has important implications for policies aimed at improving access. This study shows that nurse practitioners, who unlike physician assistants can practice independently in many states, are more likely to be located in areas of lower socioeconomic and health status than are physicians. Alternative providers such as chiropractors are 
Table 1 Median Rate of Primary Care Physicians, Nurse Practitioners, Physician Assistants, and Chiropractors According to County Socioeconomic and Health Status

\begin{tabular}{|c|c|c|c|c|}
\hline & \multicolumn{4}{|c|}{ Total or median rate, no. per 10,000 (IQR) } \\
\hline & Primary care physicians & Nurse practitioners & Physician assistants & Chiropractors \\
\hline Total no. of providers & 272,105 & 63,615 & 47,006 & 43,278 \\
\hline Overall county rate & $6.3(3.7,9.8)$ & $1.9(0.8,3.4)$ & $1.0(0.0,2.3)$ & $1.5(0.6,2.7)$ \\
\hline \multicolumn{5}{|c|}{ By socioeconomic status* } \\
\hline Quartile 1, lowest & $5.1(3.0,7.7)$ & $1.8(0.6,3.4)$ & $0.0(0.0,1.5)$ & $0.7(0.0,1.5)$ \\
\hline Quartile 2 & $6.4(3.9,9.9)$ & $2.1(0.8,3.5)$ & $1.0(0.0,2.2)$ & $1.5(0.7,2.4)$ \\
\hline Quartile 3 & $6.7(4.0,10.6)$ & $2.1(0.7,3.7)$ & $1.3(0.0,2.7)$ & $2.0(1.0,3.6)$ \\
\hline Quartile 4, highest & $7.6(4.4,10.8)$ & $1.9(0.8,3.1)$ & $1.4(0.5,2.5)$ & $2.0(1.2,3.3)$ \\
\hline \multicolumn{5}{|l|}{ By health status } \\
\hline Quartile 1, lowest & $5.2(3.2,8.1)$ & $2.1(1.0,3.7)$ & $0.4(0.0,1.4)$ & $0.8(0.0,1.4)$ \\
\hline Quartile 2 & $5.8(3.5,9.4)$ & $1.9(0.8,3.4)$ & $0.8(0.0,1.9)$ & $1.4(0.5,2.2)$ \\
\hline Quartile 3 & $7.0(4.3,10.3)$ & $2.0(0.7,3.3)$ & $1.4(0.2,2.9)$ & $2.0(1.2,3.3)$ \\
\hline Quartile 4, highest & $7.5(4.1,11.1)$ & $1.9(0.6,3.2)$ & $1.5(0.0,2.9)$ & $2.4(1.3,4.1)$ \\
\hline
\end{tabular}

$I Q R$, interquartile range

*County socioeconomic status based on median household income (in \$000s): quartile 1 (25-39), quartile 2 (39-45), quartile 3 (45-53), and quartile 4 (53-126)

County health status based on life expectancy (in years): quartile 1 (68-76), quartile 2 (76-79), quartile 3 (78-80), and quartile 4 (80-87)

A

Socioeconomic Status
Quartile 1, lowest
Quartile 2
Quartile 3
Quartile 4, highest
Health Status
Quartile 1, lowest
Quartile 2
Quartile 3
Quartile 4, highest

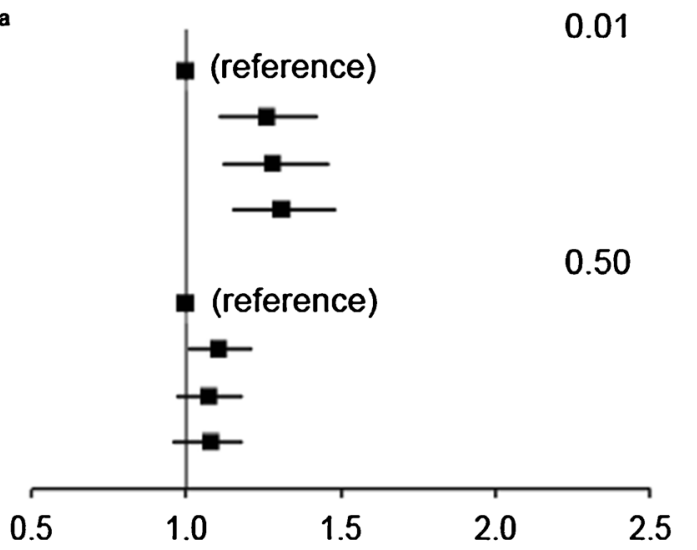

C
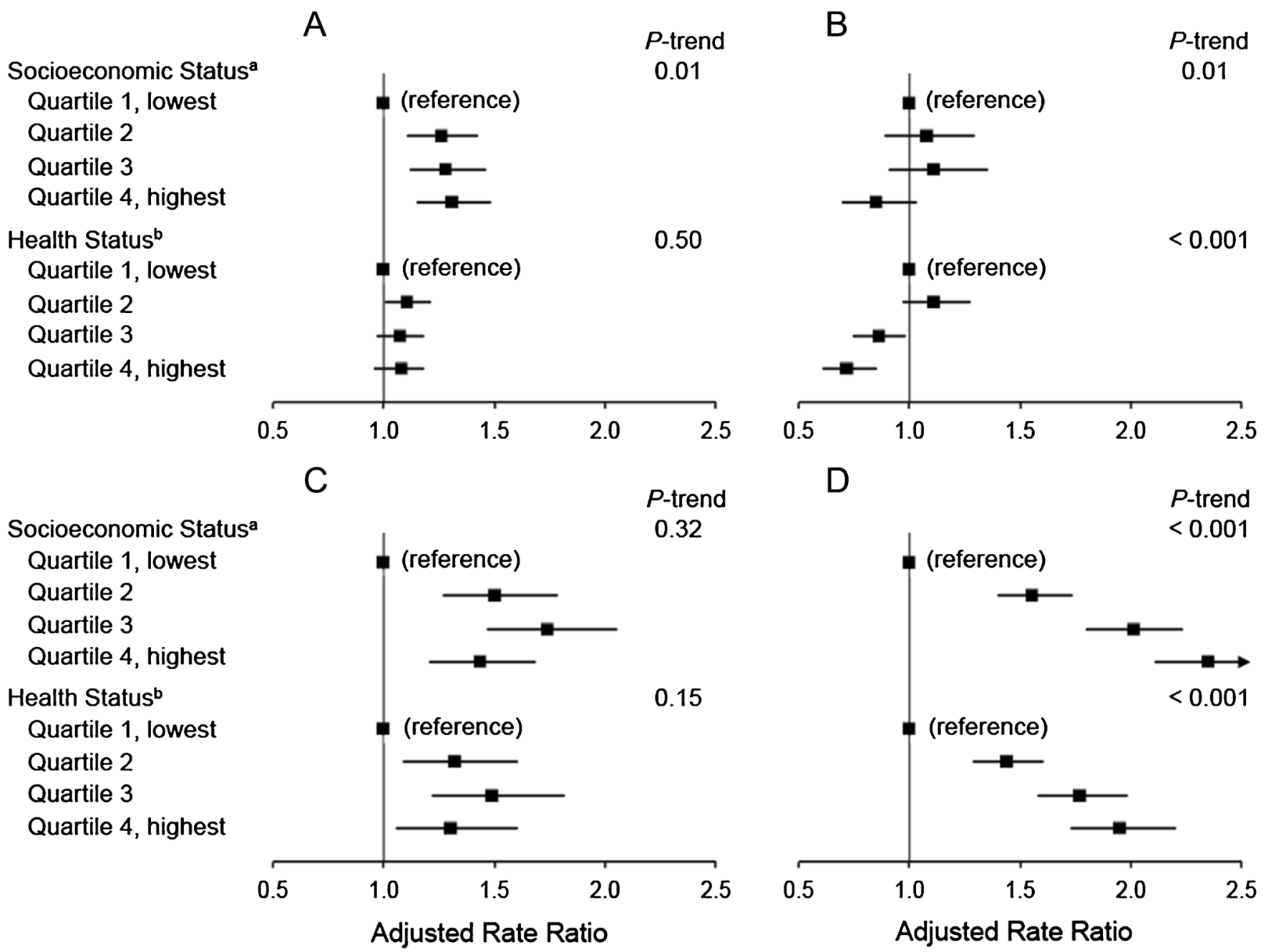

Figure 1 Adjusted rate ratios for the association between county characteristics and supply of (A) primary care physicians, (B) nurse practitioners, (C) physician assistants, and (D) chiropractors. Rate ratios adjusted for age (percentage $\geq 65$ years), sex (percentage female),

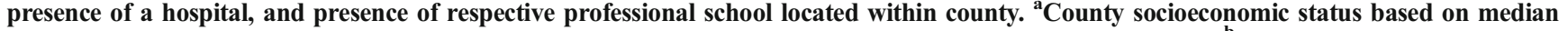

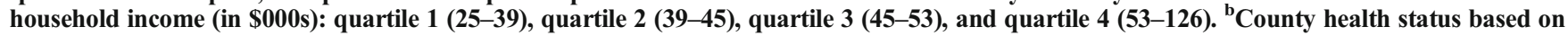
life expectancy (in years): quartile 1 (68-76), quartile 2 (76-79), quartile 3 (78-80), and quartile 4 (80-87) 
least likely to locate in these higher-need areas. Policies for enhancing access to care for underserved communities should incorporate incentives to encourage providers to locate in areas of greatest need.

Acknowledgements: This work was supported by the National Center for Complementary and Integrative Medicine at the National Institutes of Health under award number R01AT009003.

Corresponding Author: Matthew A. Davis, MPH, $\mathrm{PhD}$; University of Michigan Institute for Social Research, Ann Arbor, MI, USA (e-mail: mattadav@umich.edu).

\section{Compliance with Ethical Standards:}

Conflict of Interest: All authors declare that they have no conflict of interest.

\section{REFERENCES}

1. US Health Resource \& Services Administration. Projecting the Supply and Demand for Primary Care Practitioners through 2020. Available at: https://bhw.hrsa.gov/health-workforce-analysis/primary-care-2020. Accessed 15 Sept 2017.

2. Hooker RS, Brock DM, Cook ML. Characteristics of nurse practitioners and physician assistants in the United States. J Am Assoc Nurse Pract. 2016;28(1):39-46.

3. Rosenthal MB, Zaslavsky A, Newhouse JP. The geographic distribution of physicians revisited. Health Serv Res. 2005;40(6 Pt 1):1931-52.

4. Shipman SA, Lan J, Chang CH, Goodman DC. Geographic maldistribution of primary care for children. Pediatrics. 2011;127(1):19-27.

5. Dwyer-Lindgren L, Bertozzi-Villa A, Stubbs RW, et al. Inequalities in Life Expectancy Among US Counties, 1980 to 2014: Temporal Trends and Key Drivers. JAMA Intern Med. 2017;177(7):1003-11.

6. Starfield B, Shi L, Macinko J. Contribution of primary care to health systems and health. Milbank Q. 2005;83(3):457-502. 